\title{
Framework for conformity assessment based on an analogy with the Uncertainty Principle of Quantum Mechanics (FCAUP)
}

\author{
Celso Francisco de Moraes $^{\mathrm{a}, *}$, Messias Borges Silva $^{\mathrm{b}}$ \\ ${ }^{a}$ UNESP - Universidade Estadual Paulista, Guaratinguetá, SP, Brazil \\ ${ }^{\mathrm{b}}$ Institution: USP - University of São Paulo - School of Engineering of Lorena, Lorena, SP, Brazil
}

\section{A R T I C L E I N F O}

\section{Keywords:}

Conformity assessment

Framework

Uncertainty principle analogy

FCAUP

\begin{abstract}
A B S T R A C T
This paper aims to introduce an innovative framework, named FCAUP, for planning and implementing conformity assessment based on a conceptual analogy with Heisenberg's Uncertainty Principle of Quantum Mechanics. Conformity assessment is a very important issue in many different areas, such as, operations and quality management, international trade, sustainable development, and so on. FCAUP means "Framework for Conformity Assessment inspired by the Uncertainty Principle". This original procedure employs quantitative and qualitative techniques for analysis, classification, purposive sampling and execution of double inspections in order to reduce the uncertainty in conformity assessment activities. Its preliminary evaluation has been performed by means of three simulations regarding conformity assessments in manufacturing industries. Simulation results suggest that FCAUP is a consistent method to be used in several categories of conformity assessment.
\end{abstract}

\section{Introduction - conformity assessment review}

Conformity assessment provides several benefits to human activities, due to its importance in a wide range of aspects, such as fair competition, encouragement to quality improvement and sustainable development, environmental responsibility, customer protection, incentive for international trade, valorization of brands and products, and so on. Recent researches explain its relevance (Marsal-Llacuna, 2017; Lüth et al., 2015; Liepina et al., 2014; Theodorou and Zannikos, 2014; Assalim and Almeida, 2013). According to international standard ISO/ IEC 17000 (2004a), conformity assessment involves activities to demonstrate the fulfillment of specified requirements by products, processes, systems, personnel or bodies.

As indicated by Fig. 1, conformity assessment comprises three functions that satisfy the need or demand for demonstrating that specified requirements are fulfilled by:

- Selection;

- Determination;

- Review and attestation.

Conformity assessment typically comprises a set of specified requirements, a procedure for assessing the conformity of a product to requirements and a statement to demonstrate that requirements have been fulfilled (ISO/IEC 17000, 2004a). Furthermore, according to ISO/ IEC GUIDE 60 (2004b), there are three types of conformity assessment:

- First-party conformity assessment - activity that is performed by the person or organization that provides the object;

- Second-party conformity assessment - activity that is performed by a person or organization that has a user interest in the object;

- Third-party conformity assessment - activity that is performed by a person or body that is independent of the person or organization that provides the object.

The most common technical procedures to assess conformity in many different areas are inspection, sampling, testing, quality management system assessment and audit. Inspection is defined by ISO 9000 (2015) as "determination of conformity to specified requirements" and a similar definition is given by Juran and Godfrey (1999). Liepina et al. (2014) stated that "conformity assessment is closely related to quality management" and discussions about quality management remain relevant in several areas as pointed out by Cho et al. (2017), Li et al. (2017), Zeng et al. (2017), Rusu (2016), Siva et al. (2016), Suwandej (2015), Gerolamo et al. (2014), Wiengarten and Pagell (2012), Simon et al. (2011), among others. Therefore, there is a strong relationship among conformity assessment, quality management and inspection activities.

\footnotetext{
* Corresponding author. UNESP - Universidade Estadual Paulista, Av. Ariberto Pereira da Cunha, 333, 12516-410 Guaratinguetá, SP, Brazil.

E-mail addresses: celso.moraes@unesp.br (C.F. de Moraes), messias@dequi.eel.usp.br (M.B. Silva).
} 


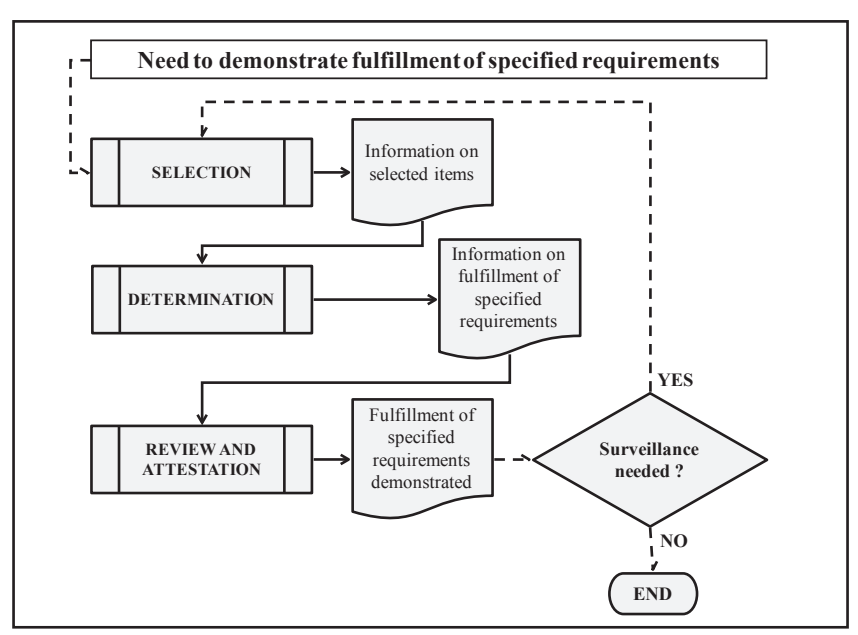

Fig. 1. Functional approach to Conformity Assessment (ISO/IEC 17000, 2004a).

The discussions presented herein refer to conformity assessment in a broad sense, however, it is focused on products and processes that present planned and regular inspection activities prior to first-party conformity assessment actions (e.g.: manufacturing industries in general).

\section{Practical considerations regarding conformity assessment}

A fundamental question that is always posed when carrying out a first-party conformity assessment is "what is the best approach to be used in order to perform a complete, useful and reliable conformity evaluation?" In other words, this issue is concerned with the establishment of an assessment plan containing a definition of extension and depth of verifications needed to determine the fulfillment of requirements under discussion. In several situations, when first-party conformity assessment is carried out after formal inspection activities, the main question is on the choice of one among three possible approaches presented in Fig. 2:
- Approach 1 (no double inspection);

- Approach 2 (complete double inspection);

- Approach 3 (partial double inspection).

Choosing the first approach is supposed to be relatively unsafe because it is based on total trust in previous inspection results. In such a case, there is no opportunity to disclose any kind of problems or mistakes during previous inspection activities. Approach 2 is certainly an appropriate option in terms of conformity assurance because a complete double inspection should be included in assessment activities. However, this option is sometimes unfeasible due to limitations on performing specific double check activities (e.g.: technical, operational, economical, chronological or physical constraints).

Regarding the third alternative, further considerations are required since it is a good technique that has been extensively used during conformity assessments. Nevertheless, if one selects approach 3, a second question must be addressed: "what is the magnitude of partial double inspection?" Its answer will define what is considered mandatory and what is considered negligible, but this is not as obvious, and it may be crucial for assessing results.

Many different methods could be employed to solve this dilemma, such as technical judgment, statistical samplings, decision making tools, selections based on identified limitations and/or empirical choices. To answer the most important questions exposed above, an innovative framework for planning and carrying out conformity assessment based on a conceptual analogy with Heisenberg's Uncertainty Principle has been developed and it is going to be presented hereafter.

\section{Uncertainty in conformity assessment}

In this paper, discussions about uncertainty are not aimed at measuring uncertainty, which is in accordance with Kuselman et al. (2017), Carobbi and Pennecchi (2016) and Pendrill (2014), just to mention a few. Differently, it is mainly focused on the uncertainty related to judgment regarding conformity assessment activities. In several situations, it is necessary to make decisions by performing a suitable examination of available conformity records, while at the same time considering the degree of trust in supposed conformity during

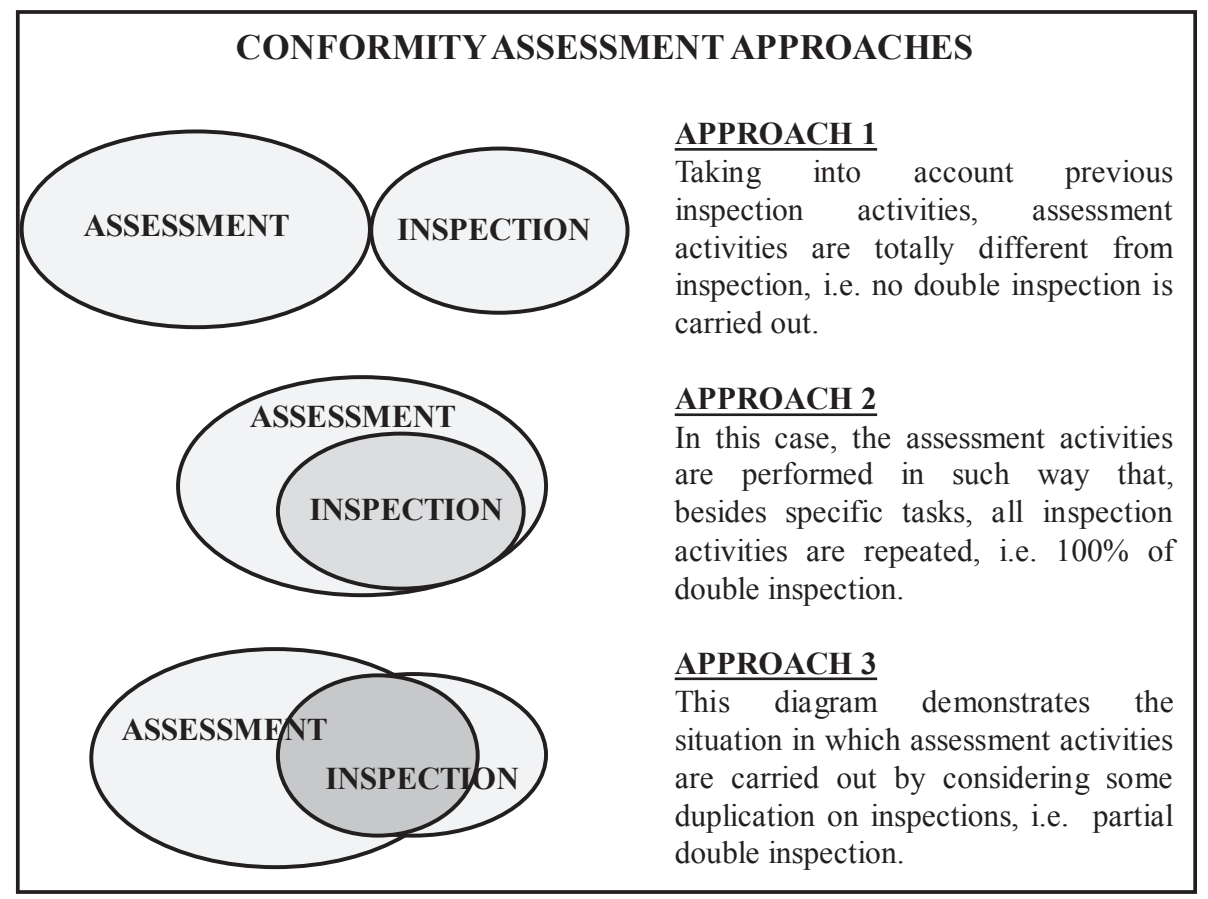

Fig. 2. Three approaches regarding the relationship between assessment and inspection. 


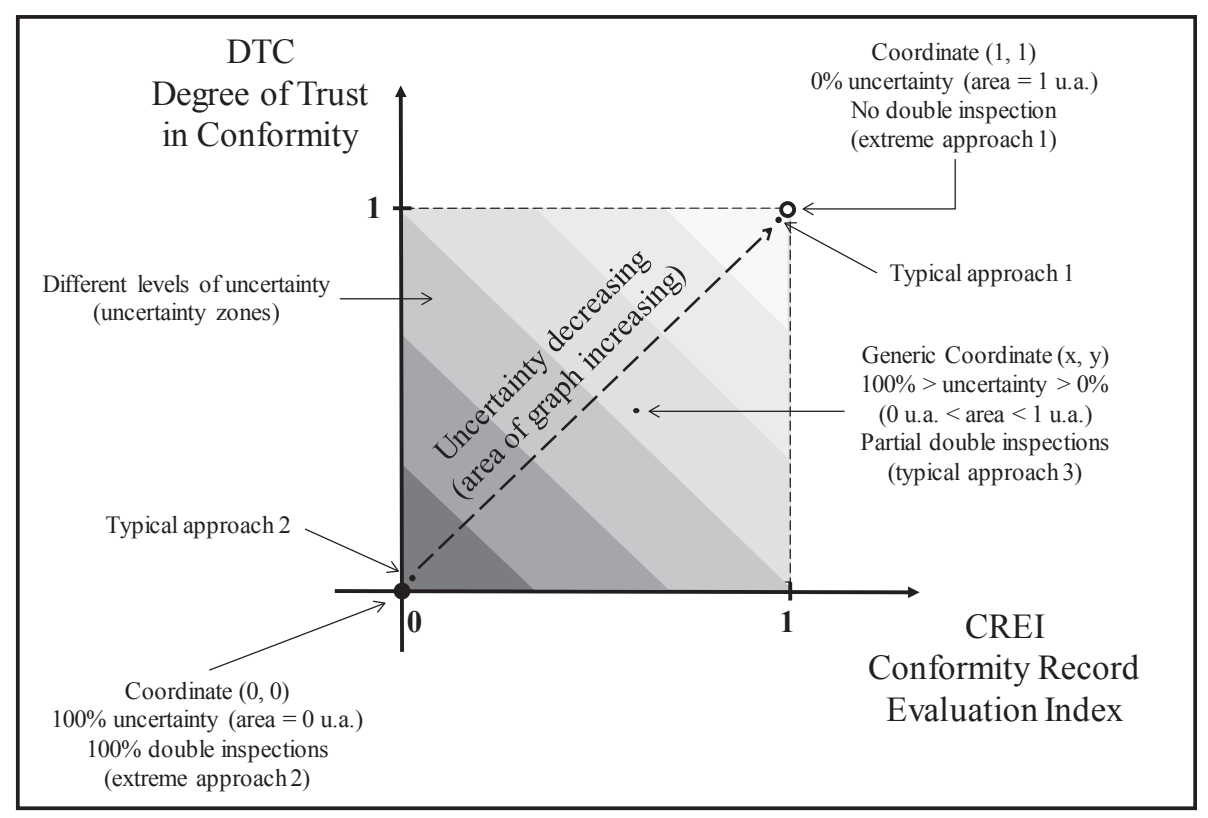

Fig. 3. Evaluation of records versus degree of trust in conformity.

assessment.

In order to formulate a systematic understanding of these situations, useful indicators of conformity records and degree of trust in conformity status have been plotted in an adapted diagram of complex numbers, also known as Argand diagram (see Fig. 3). In this correspondence with complex numbers, the objective parameter (conformity record evaluation) can be measured by the real axis (abscissas), and the subjective parameter (degree of trust in conformity) can be measured through imaginary axis (ordinates).

A white dot located at coordinates $(1,1)$ on the adapted diagram of Fig. 3 represents an ideal situation in conformity assessments in which all needed and available records have been completely appraised, while there is total and unquestionable confidence on the conformity status under discussion at the same time. This theoretical scenario does not induce any additional inspection efforts to assess conformity, i.e. no double inspection is required (see approach 1 in Fig. 2). Therefore, practical situations symbolized by a typical location which is very close to this white dot could be handled without any additional inspections due to the fact that the level of uncertainty is extremely low.

On the other hand, a black dot located at coordinate $(0,0)$ indicates the worst-case scenario in which available records have not been appraised and the degree of trust in conformity is null. This hypothetical scenario requires all possible additional inspection efforts to assess conformity, i.e. complete double inspection is necessary (see approach 2 in Fig. 2). Consequently, practical situations represented by a typical location near the aforementioned black dot must be handled by considering all possible extra inspection efforts due to a very high level of uncertainty.

Median grey zones on the diagram in Fig. 3 denote typical situations of uncertainty by considering that defining whether double inspections are indeed required is rather complex (see approach 3 in Fig. 2). In fact, all intermediate zones in the diagram represent a huge uncertainty zone at varied levels. Furthermore, from coordinate $(0,0)$, black dot, to coordinate $(1,1)$, white dot, absolute uncertainty (need to carry out complete double inspection) can be changed into total certainty (no double inspection required).

Thus, a practical quantitative correlation between uncertainty levels and zone areas on the diagram in Fig. 3 can be established: the diagram area increases as the uncertainty level decreases in the same ratio. In other words, the black dot $(0,0)$ related area is 0 units of area (u.a.) and the uncertainty level reaches the highest value $(100 \%)$, then demanding
$100 \%$ of double inspections. The white dot $(1,1)$ area is 1 u.a. and the uncertainty level reaches the lowest value $(0 \%)$, i.e., no double inspection is required. This quantitative relationship can be extrapolated to all intermediate grey zones: once there is a generic dot within an intermediate zone, it is possible to define the zone area, the remaining area, the uncertainty area or zone and, consequently, the quantitative recommendation in terms of minimum amount of double inspections to be carried out.

Obviously, there is no point in stating that a simple increase in double inspections will undoubtedly result in a complete elimination of the uncertainty inherent to the conformity assessment process. Moreover, as indicated in the rationale of Fig. 2, in many practical situations there are technical, operational, economic, chronological or physical constraints that prevent the implementation of double checks. Nevertheless, whenever applicable, double inspection is still an effective tool used to reduce the uncertainty level associated to the conformity assessment activities.

In order to create both realistic and useful diagrams, additional considerations regarding CREI (conformity record evaluation index), at axis of abscissas, and DTC (degree of trust in conformity), at axis of ordinates, must be presented. An examination of available records can be translated into an objective parameter by using, for instance, the indicator presented in Equation (1).

CREI $=\frac{\text { Qty }_{\text {evaluated }}}{\text { Qty }}$

Where:

$C R E I=$ conformity record evaluation index;

Qty $_{\text {evaluated }}=$ actual quantity of records evaluated in detail;

Qty $_{\text {total }}=$ total quantity of available records.

Accordingly, the axis of abscissas can be represented by a continuous variable $(0 \leq \mathrm{CREI} \leq 1)$, which is perfectly coherent with the main idea in Fig. 3. Nevertheless, the degree of trust in the assumed conformity status is a subjective parameter since it is biased due to personal feelings or opinions. Consequently, a reasonable way to represent it on the axis of ordinates in the diagram is by means of attributes, instead of a continuous variable.

Low and high are the simplest attributes that can be associated with the degree of trust in conformity (DTC) at axis of ordinates. However, 


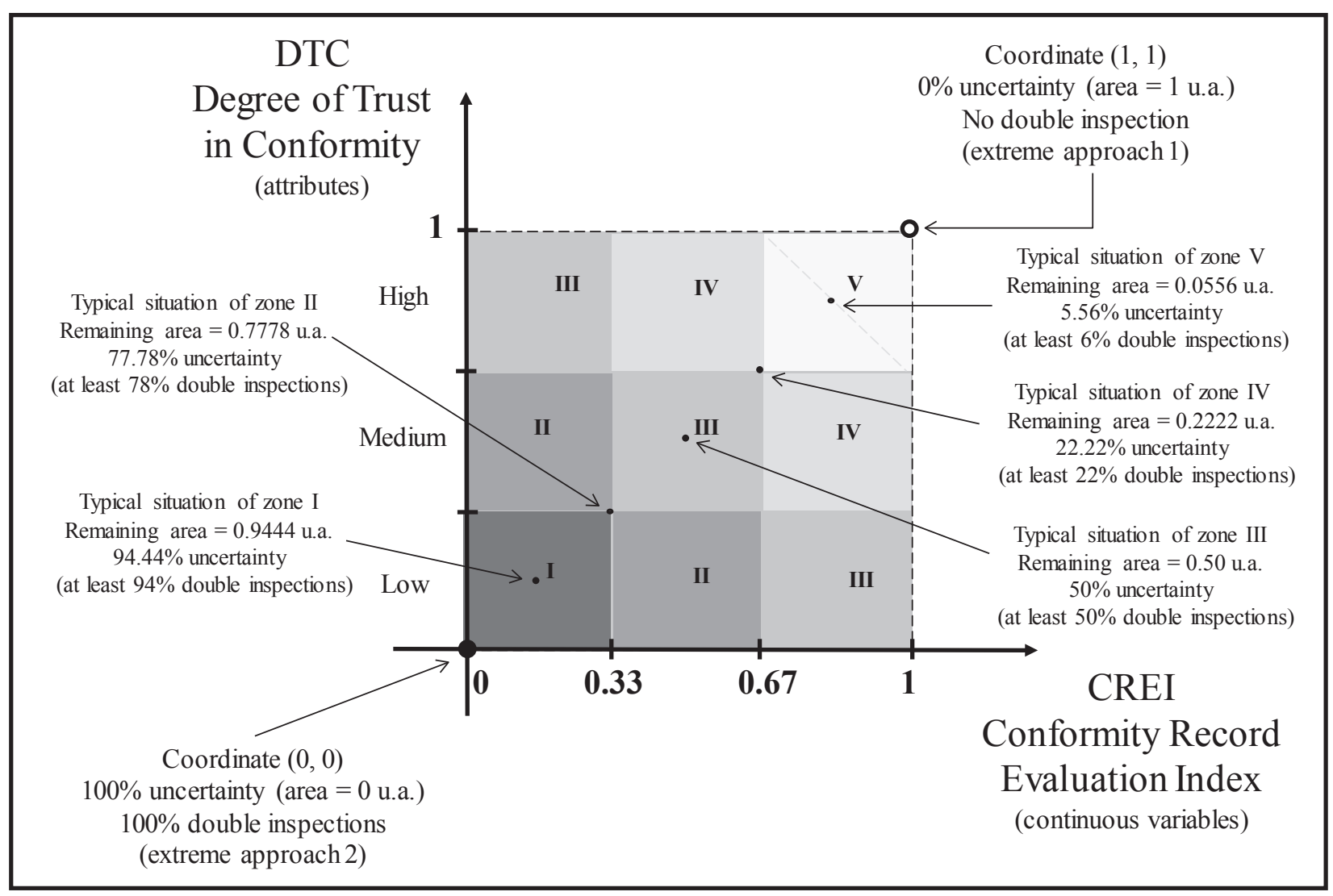

Fig. 4. Evaluation of records versus degrees of trust (low, medium and high).

only two attributes (low and high) may be incomplete to represent the degrees of trust in conformity and, moreover, in this specific case, a pair of attributes does not allow including an intermediary attribute, e.g. medium. Therefore, three attributes, low, medium and high, are more representative about the degree of trust in conformity (DTC) at axis of ordinates.

Fig. 4 illustrates the adjusted diagram configuration when three attributes (low, medium and high) on the axis of ordinates are associated with variables on the axis of abscissas. For purposes of symmetry, the axis of abscissas must also be divided into three parts or intervals (0-0.33, 0.33 to 0.67 and 0.67 to 1 ), thus nine segments emerge and can be suitably grouped into five different zones (I, II, III, IV and V).

Areas of the five uncertainty zones depicted in Fig. 4 have been calculated by considering intermediate points as representative and typical of those zones. See detailed calculation regarding five uncertainty zones shaped by three degrees of trust in Table 1 .

The quantitative suggestions with respect to minimum double inspections defined in Fig. 4 and Table 1 by five zones ( $94 \%$ and $78 \%$ for pessimistic situations, $50 \%$ for intermediary situations, $22 \%$ and $6 \%$ for optimistic situations) are properly combined in Table 2 as a decision matrix. This decision matrix presents three degrees of trust in conformity (DTC), three intervals of conformity record evaluation index (CREI) and nine segments grouped into five uncertainty zones.

The situation with three degrees of trust and five uncertainty zones as presented until now by means of Fig. 4 and Table 2 is supposed to be appropriate to describe the uncertainty levels under discussion in terms of conformity assessment. Nevertheless, there are conditions to extrapolate the discussions regarding this kind of uncertainty, that is, it is possible to increase the quantity of attributes associated with the degree of trust and, consequently, the uncertainty zones and the quantitative recommendation for double inspections.

For instance, bearing in mind the diagram of Fig. 3, five attributes (very low, low, medium, high and very high) on the axis of ordinates can be associated with variables on the axis of abscissas. Once more, for purposes of symmetry, the axis of abscissas must also be divided into five parts or intervals $(0-0.2,0.2$ to $0.4,0.4$ to $0.6,0.6$ to 0.8 and 0.8 to 1 ), thus these twenty-five segments can be grouped into nine different zones (I, II, III, IV, V, VI, VII, VIII and IX). See detailed calculation regarding nine uncertainty zones shaped by five degrees of trust in Table 3.

Table 1

Calculation of areas regarding 5 uncertainty zones shaped by 3 degrees of trust.

\begin{tabular}{|c|c|c|c|c|}
\hline Zone & $\begin{array}{l}\text { Zone area } \\
\text { (u.a) }\end{array}$ & Remaining area (total area minus all previous areas) & $\begin{array}{l}\text { Uncertainty zones (half of zone area plus } \\
\text { remaining area) }\end{array}$ & $\begin{array}{l}\text { Recommendation of minimum } \% \text { of double } \\
\text { inspection required }\end{array}$ \\
\hline I & 0.1111 & $0.8889(1-0.1111)$ & $0.9445(0.1111 / 2+0.8889)$ & $94 \%$ \\
\hline II & 0.2222 & $0.6667(1-0.1111-0.2222)$ & $0.7778(0.2222 / 2+0.6667)$ & $78 \%$ \\
\hline III & 0.3333 & $0.3334(1-0.1111-0.2222-0.3333)$ & $0.5000(0.3333 / 2+0.3334)$ & $50 \%$ \\
\hline IV & 0.2222 & $0.1112(1-0.1111-0.2222-0.3333-0.2222)$ & $0.2223(0.2222 / 2+0.1112)$ & $22 \%$ \\
\hline $\mathrm{V}$ & 0.1111 & $0.0000(1-0.1111-0.2222-0.3333-0.2222-0.1111)$ & $0.0556(0.1111 / 2+0.0000)$ & $6 \%$ \\
\hline
\end{tabular}


Table 2

Recommendation of double inspections (decision matrix with 3 degrees of trust).

\begin{tabular}{|c|c|c|c|c|}
\hline \multicolumn{2}{|l|}{ Recommendation of Double Inspections } & \multicolumn{3}{|c|}{ CREI (conformity record evaluation index) } \\
\hline & & $0 \leq \mathrm{CREI} \leq 0,33$ & $0,33<\mathrm{CREI}<0,67$ & $0,67 \leq \mathrm{CREI} \leq 1$ \\
\hline \multirow[t]{3}{*}{ DTC (degree of trust in conformity) } & High & $50 \%$ (Zone III) & $22 \%$ (Zone IV) & $6 \%$ (Zone V) \\
\hline & Medium & $78 \%$ (Zone II) & $50 \%$ (Zone III) & $22 \%$ (Zone IV) \\
\hline & Low & $94 \%$ (Zone I) & $78 \%$ (Zone II) & $50 \%$ (Zone III) \\
\hline
\end{tabular}

Table 3

Calculation of areas regarding 9 uncertainty zones shaped by 5 degrees of trust.

“CREI X DTC” with 5 degrees of trust (very low, low, medium, high, very high) and 9 uncertainty zones (I to IX)

\begin{tabular}{lllll}
\hline Zone & $\begin{array}{l}\text { Zone } \\
\text { area } \\
\text { (u.a) }\end{array}$ & $\begin{array}{l}\text { Remaining area } \\
\text { (total area } \\
\text { minus all } \\
\text { previous areas) }\end{array}$ & $\begin{array}{l}\text { Uncertainty zones } \\
\text { (half of zone area } \\
\text { plus remaining } \\
\text { area) }\end{array}$ & $\begin{array}{l}\text { Recommendation of } \\
\text { minimum \% of double } \\
\text { inspection required }\end{array}$ \\
\hline I & 0.04 & 0.96 & 0.98 & $98 \%$ \\
II & 0.08 & 0.88 & 0.92 & $92 \%$ \\
III & 0.12 & 0.76 & 0.82 & $82 \%$ \\
IV & 0.16 & 0.60 & 0.68 & $68 \%$ \\
V & 0.20 & 0.40 & 0.50 & $50 \%$ \\
VI & 0.16 & 0.24 & 0.32 & $32 \%$ \\
VII & 0.12 & 0.12 & 0.18 & $18 \%$ \\
VIII & 0.08 & 0,04 & 0.08 & $8 \%$ \\
IX & 0.04 & 0.00 & 0.02 & $2 \%$ \\
\hline
\end{tabular}

The quantitative suggestions with respect to minimum double inspections defined in Table 3, from zone I ( $98 \%$ of double inspections, the most pessimistic) to zone IX ( $2 \%$ of double inspections, the most optimistic) are properly combined in Table 4 as a decision matrix with five degrees of trust in conformity (DTC), five intervals of conformity record evaluation index (CREI) and twenty-five segments grouped into nine uncertainty zones. Once again, the intermediary zone (V) indicates $50 \%$ of double inspections as a quantitative recommendation.

Based on the considerations and calculations presented so far, one may extrapolate the quantity of attributes regarding the degree of trust in conformity and the resulting uncertainty zones of this adapted diagram by using the formula provided by Equation (2).

Qty $y_{\text {zones }}=2 \times$ Qty trust deg -1

Where:

Qty $_{\text {zones }}=$ quantity of uncertainty zones plotted on the adapted Argand diagram;

Qty $_{\text {trust deg }}=$ quantity of attributes regarding the degrees of trust in conformity.

For instance, the use of seven degrees of trust in conformity will create thirteen uncertainty zones $(2 \times 7-1=13)$. In this way, if one decides to establish seven degrees of trust (e.g.: extremely low, very low, low, medium, high, very high and extremely high), and based on the symmetry of diagram of Fig. 3, forty-nine segments will be grouped into thirteen zones. Accordingly, it is possible to define a recommendation in terms of minimum double inspections to be carried out by considering each zone from the most pessimistic scenario (zone I: $99 \%$ of double inspections) to the most optimistic scenario (zone XIII: $1 \%$ of double inspections). Once more, the intermediary zone (VII) indicates $50 \%$ of double inspections as a quantitative recommendation. In this case, a suitable decision matrix considering seven degrees of trust in conformity may be arranged.

It might be assumed that over seven degrees of trust could make it difficult to be satisfactorily defined. Furthermore, more than thirteen uncertainty zones are not so useful in practical terms. Therefore, by considering the discussions presented so far, an odd number which is greater than two, but not exceeding seven, i.e., 3, 5 or 7 , should be a reasonable suggestion for establishing the amount of degrees of trust in conformity.

The recommendation for double inspections based on uncertainty zones of adapted diagrams presented herein is strictly quantitative (minimum amount in percentage terms) and, consequently, there is no qualitative connotation. A qualitative analysis is relatively complex because it is sometimes circumscribed by many conditions that cannot be properly recognized and/or controlled. Thus, any recommendation for double inspection should be made by firstly considering the quantitative analysis (adapted diagram areas) and secondly by a qualitative investigation that can be based on different criteria or ranking methods, such as, technical, operational or economical judgment; physical or chronological feasibility; common sense; random selection, and so on.

\section{Brief review of Quantum Mechanics}

According to Shankar (1994), the expanding domain of Classical Physics encountered its first obstacles around the beginning of the twentieth century. It has arisen on two fronts: at large velocities and small scales. The problem of large velocities was successfully solved by Relativistic Mechanics, while Quantum Mechanics solved the problem of small scales.

Quantum Mechanics or Quantum Physics is a branch of Physics which explains physical phenomena at small scales or, as detailed by Fitts (2002), entities such as atoms, electrons, protons, neutrons, atomic nuclei, as well as photons. Its theory has been developed during the first half of the twentieth century through efforts of many scientists, such as Max Planck (1858-1947), Albert Einstein (1879-1955), Max Born (1882-1970), Neils Bohr (1885-1962), Erwin Schrödinger (1887-1961), Louis de Broglie (1892-1987), Wolfgang Pauli (1900-1958), Werner Heisenberg (1901-1976), Paul Dirac

Table 4

Recommendation of double inspections (decision matrix with 5 degrees of trust).

\begin{tabular}{|c|c|c|c|c|c|c|}
\hline \multicolumn{2}{|l|}{ Recommendation of Double Inspections } & \multicolumn{5}{|c|}{ CREI (conformity record evaluation index) } \\
\hline & & $0 \leq \mathrm{CREI}<0.2$ & $0.2 \leq \mathrm{CREI}<0.4$ & $0.4 \leq \mathrm{CREI}<0.6$ & $0.6 \leq \mathrm{CREI}<0.8$ & $0.8 \leq \mathrm{CREI} \leq 1$ \\
\hline \multirow[t]{5}{*}{ DTC (degree of trust in conformity) } & Very high & $50 \%$ (Zone V) & $32 \%$ (Zone VI) & $18 \%$ (Zone VII) & $8 \%$ (Zone VIII) & $2 \%$ (Zone IX) \\
\hline & High & $68 \%$ (Zone IV) & $50 \%$ (Zone V) & $32 \%$ (Zone VI) & $18 \%$ (Zone VII) & $8 \%$ (Zone VIII) \\
\hline & Medium & $82 \%$ (Zone III) & $68 \%$ (Zone IV) & $50 \%$ (Zone V) & $32 \%$ (Zone VI) & $18 \%$ (Zone VII) \\
\hline & Low & $92 \%$ (Zone II) & $82 \%$ (Zone III) & $68 \%$ (Zone IV) & $50 \%$ (Zone V) & $32 \%$ (Zone VI) \\
\hline & Very low & $98 \%$ (Zone I) & $92 \%$ (Zone II) & $82 \%$ (Zone III) & $68 \%$ (Zone IV) & $50 \%$ (Zone V) \\
\hline
\end{tabular}


(1902-1984), John von Neumann (1903-1957), Richard Feynman (1918-1988), among others.

German physicist and mathematician Max Born coined the term "Quantum Mechanics" in 1924 (Fedak and Prentis, 2009) in reference to a revolutionary work published by Max Planck in 1900, in which it has been proposed that some physical quantities can change only in discrete amounts (quanta from Latin language) and not continuously. Researches of Quantum Physics have demonstrated the inadequacy of Classical Physics in explaining some phenomena related to small particles. According to those researches, it appears that each particle has associated with it a wave function providing only the probability of finding it at a point $x$ at time $t$. This is called wave-particle duality. In accordance with Griffiths (2011) "all Quantum Mechanics has to offer is statistical information about the possible results and this indeterminacy has been profoundly disturbing to physicists and philosophers alike".

The Uncertainty Principle of Quantum Mechanics was published in 1927 by the German physicist Werner Heisenberg and it states that the more precisely the position of some particle is determined, the less precisely its momentum can be known, and vice versa. The Heisenberg's Uncertainty Principle is summarized by means of Equation (3) (Griffiths, 2011).

$\sigma_{x} \cdot \sigma_{p} \geq \frac{\hbar}{2}$

Where:

$\sigma_{x}=$ standard deviation of particle's position;

$\sigma_{p}=$ standard deviation of particle's momentum (product of mass and velocity);

$\hbar=$ reduced Planck constant $\left(\hbar=\mathrm{h} / 2 \pi ; \mathrm{h}=6626 \cdot 10^{-34} \mathrm{~J} \mathrm{~s}\right)$.

Heisenberg's Uncertainty Principle is a consequence of the assumption that a quantum particle is a wave packet. This situation contrasts with classical-mechanical behavior, where both the position and momentum can, in principle, be specified simultaneously as precisely as one wishes (Fitts, 2002). According to Zozor et al. (2008), the Uncertainty Principle is a fundamental concept that attracts a great deal of attention, not only in quantum physics, but also in other areas. However, Jijnasu (2016) affirmed that the clarity that has dawned upon physicists over the decades regarding quantum uncertainty remained mostly imperceptible for general readers, students, philosophers and even non-expert scientists.

\section{Framework for Conformity Assessment inspired by the Uncertainty Principle (FCAUP)}

Bearing in mind the classical concepts of particle (very small piece of matter) and wave (disturbance or variation which travels through a medium) and recognizing the unquestionable importance of documented records and evidences during conformity assessment activities, it is necessary to establish some new definitions based on an analogy with some physical notions:

- Particle-like records (PLR) are those that can be double-checked during first-party conformity assessments without any kind of disturbance on the product under assessment;

- Wave-like records (WLR) are those in which double inspections are not feasible during first-party conformity assessments or they can cause some level of disturbance on the product under assessment;

- Position of record in conformity assessment is its objective and/or absolute information to be double-checked;

- Momentum of record in conformity assessment is its subjective and/or interpretative evaluation/verification based on available information.

In accordance with these definitions, during the initial approach of a conformity assessment, a particle-like record (PLR) seems to allow suitable double inspection on its position whereas its momentum is not as useful. On the other hand, it seems to be difficult (or even impossible) to perform double inspection on the position of a wave-like record (WLR), but its momentum may be relatively useful.

This initial approach of a conformity assessment resembles the conventional interpretation of macroscopic phenomena given by Classical Physics. However, as conformity assessment activities evolve, and detailed qualitative observations take place, the understanding of the initial approach may not explain the actual condition of the product under assessment. Similar situation occurs when Classical Physics attempts to explain physical phenomena at subatomic scales.

The divergences between Classical Physics and Quantum Mechanics emerge as the scales decrease, that is, the quantum behavior, the waveparticle duality and the influence of the observer on the measurement process are understandable at very small scales. In the same way, during the conformity assessment activities, as the qualitative aspects are truly investigated, findings that can mix up the judgements initially established may arise and the influence of the observer, in this case the assessor, cannot be ignored (a quantum-like behavior).

During conformity assessment activities, the discrepancies between an initial approach and actual findings emerge as the qualitative investigation advances. Analogously, the extremely small scale of the Uncertainty Principle corresponds to the extremely detailed qualitative investigation in conformity assessment (see schematic representation in Fig. 5).

Based on analysis of Fig. 5, one may consider that physical measurements at subatomic scales resemble in-depth qualitative evaluations, as well as, small particles and discrete records may be assumed as analogous elements in this conceptual discussion:

- Particles have position and momentum, whereas records have also position (objective information to be double-checked) and momentum (subjective/interpretative evaluation);

- Position and momentum of particles are well-defined at macroscopic scales, but they cannot be mutually measured with absolute precision at subatomic scales, whereas presumed position and momentum of records during initial approach may change and be mixed up due to qualitative nuances of findings;

- Wave-particle duality at subatomic scales can be associated to the possible changes in qualitative interpretation and judgements as indepth investigation evolves;

- The observer effect due to quantum behavior at subatomic scales can be compared to the obvious impact caused by a qualified assessor during in-depth qualitative evaluations, that is, there is also an assessor effect caused by his/her personal interpretation of findings;

- These peculiarities of conformity assessment may be described as a quantum-like behavior.

Nevertheless, it is not the purpose of this study to demonstrate that there is mathematical connection among all postulates of the Uncertainty Principle (UP) and all characteristics of Conformity Assessment (CA). Instead, the main idea is to advocate that the knowledge of a conceptual analogy satisfactorily consistent between UP and CA can be useful in establishing structured activities to support and simplify the execution of conformity assessment.

The central proposition of this paper, i.e. a conceptual analogy between the Uncertainty Principle (UP) and Conformity Assessment (CA) is summarized in Table 5 by means of five topics. Based on that analogy, an original framework for planning and implementing conformity assessment by considering approach 3 (partial double inspection) has now been introduced by the acronym FCAUP (Framework for Conformity Assessment inspired by the Uncertainty Principle).

It should be observed that the acronym FCAUP is also related to the first letters of the five topics highlighted in Table 5 (Fact, Context, 


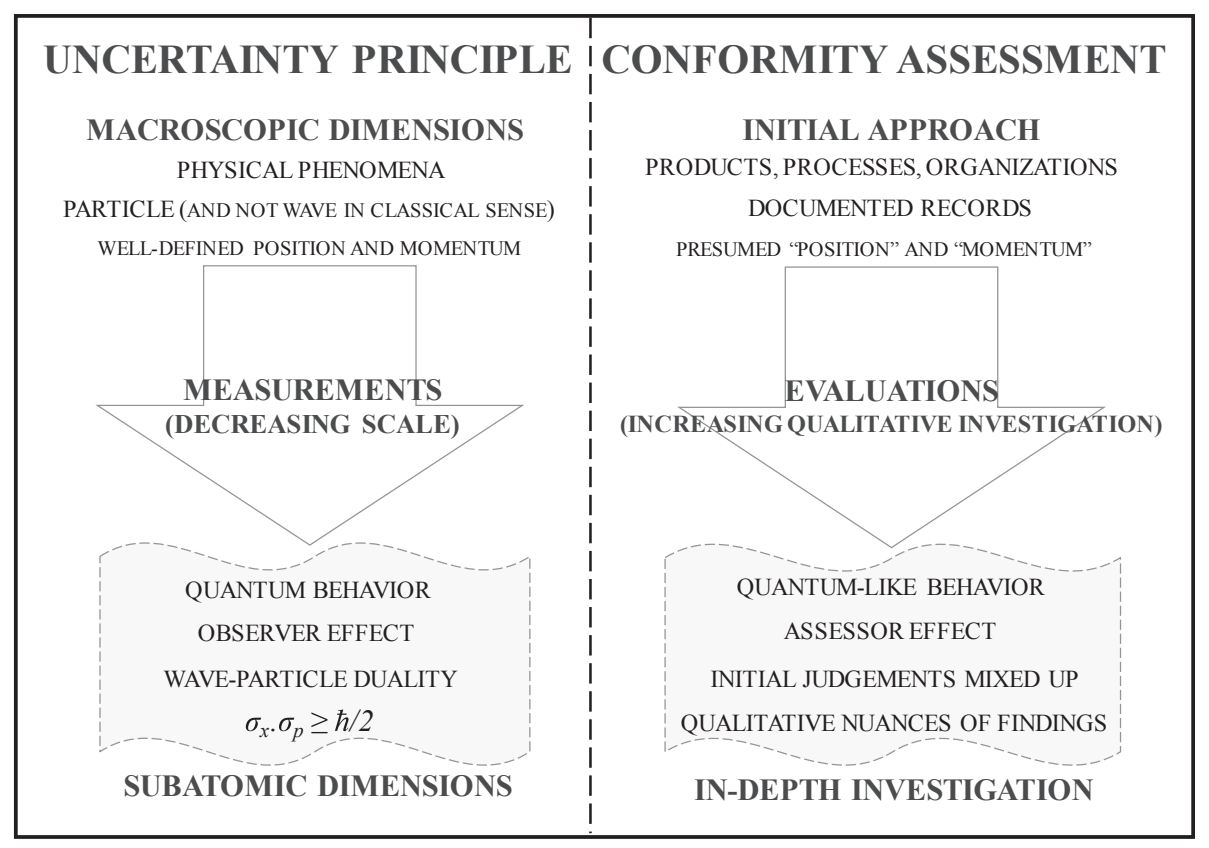

Fig. 5. Analogy between uncertainty principle and conformity assessment (diagram).

Actions, Understanding and Paradigm).

Basically, the proposed framework is a structured deployment of three functions (selection, determination, review and attestation) of conformity assessment defined by international standard ISO/IEC 17000:2004a (Fig. 1) and it is divided into five stages (Foreword, Classification, Arrangement, Uncovering and Paperwork) correlated to acronym FCAUP as well. The stages, steps, comments and instructions of the proposed procedure called as FCAUP are arranged in a flow chart shown in Fig. 6.

\section{Preliminary evaluation of FCAUP}

FACUP has been evaluated by means of three simulations based on actual examples of conformity assessment which were previously carried out by using products of different manufacturing industries: an electro-electronic equipment (industry A), a hydro-mechanical equipment (industry B) and a test set-up (industry C). Industries A, B and C have been chosen because all actual conformity records were available for the authors examination during the study. Those conformity assessments were previously carried out in December/2013, March/2014 and July/2015, respectively, without using this framework. In order to ensure confidentiality aspects, names of companies, geographical locations and details of products have been omitted.

Some of the actual records appraised during conformity assessments in Industry A (electro-electronic equipment) and Industry B (hydromechanical equipment) are shown in Fig. 7, but specific confidential details have been intentionally omitted.

To implement a preliminary evaluation of FCAUP, all actual data and records have been reexamined by considering instructions and calculations predicted by the framework. Table 6 compares the results of actual conformity assessments and those of simulated conformity assessments based on data obtained from industries A, B and C. All definitions previously presented, such as PLR (particle-like records), WLR (wave-like records), CREI (conformity record evaluation index), DTC (degree of trust in conformity; in these simulations, high, medium or low) and uncertainty zones (in these simulations, from I to V) are essential to understand Table 6.

Supplementary information explains the data presented in Table 6:

- Those actual conformity assessments have been carried out by an experienced assessor and the simulations have been performed by the authors of this study;

- During the actual assessments previously performed in industries A, $\mathrm{B}$ and $\mathrm{C}$, no specific rule has been used by the assessor to define the percentage of double inspections. Instead, the percentages were not previously defined, since they were consequence of empirical process (the values $50 \%, 75 \%$ and $30 \%$ are approximations for the actual amounts);

Table 5

Analogy between the uncertainty principle and conformity assessment (summary).

\begin{tabular}{|c|c|c|}
\hline Topic & Uncertainty principle & Conformity assessment \\
\hline Fact & Measurement of conjugate variables position (x) and momentum (p). & Determination of fulfilling specified requirements. \\
\hline Context & Physical phenomena at subatomic scales. & Products, processes, systems, personnel or organizations. \\
\hline Actions & $\begin{array}{l}\text { Advanced experiments in laboratory based on specific postulates and } \\
\text { mathematical formalism. }\end{array}$ & $\begin{array}{l}\text { Inspection, sampling, testing, quality management system assessment, audit } \\
\text { (double-checks are included). }\end{array}$ \\
\hline Understanding & $\begin{array}{l}\text { Position (x) and momentum (p) are canonically conjugate variables. The more } \\
\text { precisely one is defined, the less precisely is the other, in accordance with the } \\
\text { equation } \sigma x . \sigma p \geq \hbar / 2 \text {. }\end{array}$ & $\begin{array}{l}\text { Double-checked records have also position (objective information) and } \\
\text { momentum (subjective evaluation) and, as in-depth investigation evolves } \\
\text { during the execution of conformity assessment, the initial judgement may be } \\
\text { mixed up due to qualitative nuances of findings (quantum-like behavior). }\end{array}$ \\
\hline Paradigm & $\begin{array}{l}\text { The Uncertainty Principle is indeed related to wave-particle duality. } \\
\text { Furthermore, the experimental process itself influences the measurement } \\
\text { result due to a quantum behavior. }\end{array}$ & $\begin{array}{l}\text { There is some uncertainty in carrying out conformity assessment caused by a } \\
\text { quantum-like behavior and the assessment result is also influenced by the } \\
\text { observer (assessor). The boundaries where uncertainty does not degrade } \\
\text { assessment results may be established by means of a qualitative trade-off. }\end{array}$ \\
\hline
\end{tabular}




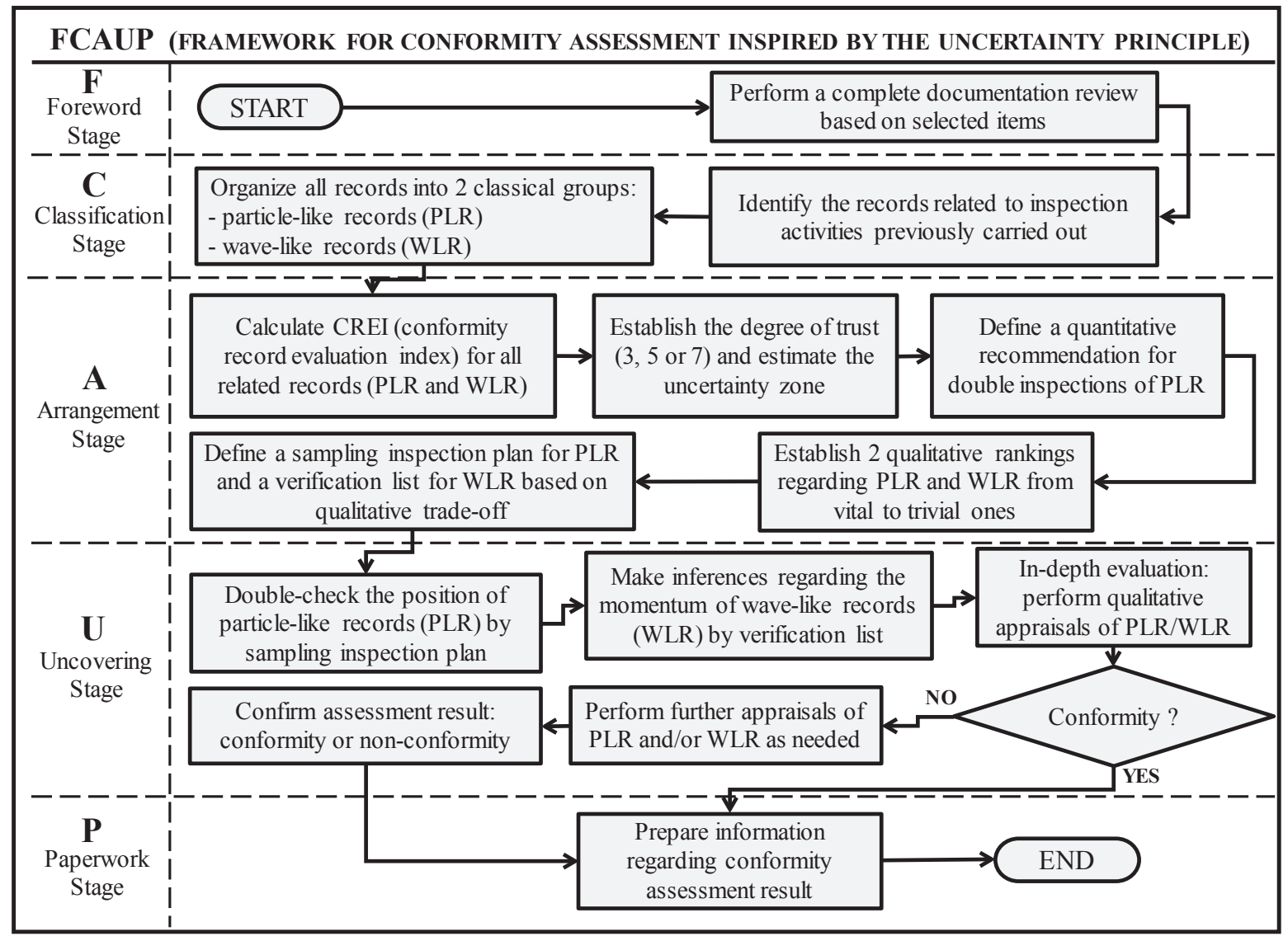

Fig. 6. FCAUP (schematic representation as a flow chart).

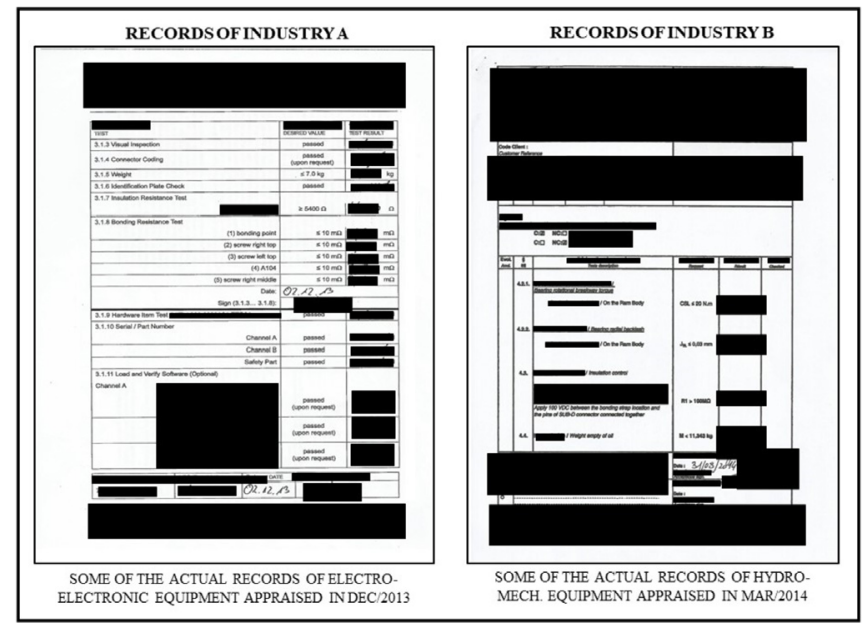

Fig. 7. Some of the actual records of Industries A and B.

- For these three simulations, all records previously collected during the actual assessments have been reviewed and divided into two categories, PLR (particle-like records) and WLR (wave-like records): electro-electronic equipment of Industry A (42 records $=34$ PLR +8 WLR), hydro-mechanical equipment of Industry B (48 records $=36$ PLR $+12 \mathrm{WLR}$ ) and test Set-up of Industry C (145 records $=25$ PLR +120 WLR);

- Some examples of particle-like records (PLR) are basic dimensions of the product, total weight, bonding resistance tests and verification of external damages, because they could be double-checked without disturbance on the product under this simulated assessment (electro-electronic equipment of Industry A);
- Some examples of wave-like records (WLR) are proof pressure and sealing test, valve functioning test, actuator stroke test under specific conditions and leakage test after $24 \mathrm{~h}$, because they could cause some disturbance on the product under this simulated assessment (hydro-mechanical equipment of Industry B);

- All available records have been evaluated by the authors of this study during the simulations and, therefore, the value for CREI (conformity record evaluation index) is 1 in the three cases as per Equation (1);

- Degrees of trust in conformity (DTC medium for industry A, low for industry B and high for industry $\mathrm{C}$ ) have been provided by the assessor responsible by the actual assessment based on his professional expertise;

- Uncertainty zones (IV for industry A, III for industry B and V for industry $\mathrm{C}$ ) have been defined by the authors in accordance with Fig. 4 and Table 2;

- Recommendations for double inspections (22\% for industry A, 50\% for industry $\mathrm{B}$ and $6 \%$ for industry $\mathrm{C}$ ) have been obtained by the authors according to Fig. 4 and Table 2;

- Quantities of double-checked PLR have been obtained by the authors considering the percentages of recommendations given by Table 2 (e.g.: $22 \%$ of $34=7,48$ or approximately $8 ; 50 \%$ of $36=18 ; 6 \%$ of $25=1,5$ or approximately 2 );

- Quantities of verified WLR have been jointly defined by the authors and assessor by means of qualitative appraisal predicted by the framework.

As informed by the assessor, specifically in the case of actual conformities carried out in Industry A and Industry B, there was a little change between the initial judgment and the understanding due to the findings after in-depth investigation. By the other hand, no change has been observed in the actual conformity assessment in industry $\mathrm{C}$ (test 
Table 6

Preliminary evaluation of FCAUP: actual assessments versus simulated assessments.

\begin{tabular}{|c|c|c|c|}
\hline & Industry A & Industry B & Industry C \\
\hline Item under assessment & Electro-electronic equipment & Hydro-mechanical equipment & Test set-up \\
\hline Date of actual assessment & Dec/2013 & Mar/2014 & Jul/2015 \\
\hline Total Qty of conformity records & 42 & 48 & 145 \\
\hline$\%$ of double inspections & $50 \%$ & $75 \%$ & $30 \%$ \\
\hline Actual assessment result & Conformity stated & Conformity stated & Conformity stated \\
\hline Date of simulation & Apr $/ 2017$ & $\mathrm{Apr} / 2017$ & $\mathrm{Apr} / 2017$ \\
\hline Qty of PLR (particle-like records) & 34 & 36 & 25 \\
\hline Qty of WLR (wave-like records) & 8 & 12 & 120 \\
\hline CREI (conf. record evaluat. index) & 1 & 1 & 1 \\
\hline DTC (degree of trust in conformity) & Medium & Low & High \\
\hline Uncertainty zone (I to V) in accordance with Fig. 4 & IV & III & $\mathrm{V}$ \\
\hline Recomm. for double insp.(\%PLR) as per decision matrix (Table 2) & $22 \%$ & $50 \%$ & $6 \%$ \\
\hline Qty of double-checked PLR & 8 & 18 & 2 \\
\hline Qty of verified WLR & 6 & 10 & 20 \\
\hline Simulated assessment result & Conformity endorsed & Conformity endorsed & Conformity endorsed \\
\hline
\end{tabular}

set-up) in terms of initial approach versus qualitative nuances. This means that it is not always that a quantum-like behavior arises during conformity assessment, but this possibility cannot be ignored.

The simulations have indicated that the use of this framework during conformity assessment is indeed advantageous because realistic results could have been reached in an improved and structured procedure. For all simulated situations, it can be observed that the percentage of double inspections is lower than that of double inspections performed during actual assessment. This happens mainly because the following key aspects of FCAUP:

- Extensive examination of available conformity records;

- Degree of trust in conformity is also considered;

- All available records are categorized;

- Some uncertainty is admitted, but its level is estimated to define countermeasures;

- Both quantitative and qualitative analyses are carried out;

- Use of non-probability sampling (purposive) based on qualitative trade-off;

- Objective inspections (position) are merged with subjective verifications (momentum);

- There is also an opportunity to perform further appraisal in case of remaining uncertainty.

In order to provide support as regards using the framework, suitable spreadsheets can be used as needed; however, they shall clearly include instructions and calculations predicted by the method FCAUP in such a way that anyone is able to properly input data. Simulations presented in Table 6 have been plotted in spreadsheets that were previously prepared for the concepts described herein.

English philosopher and theologian William of Ockham (1285-1349) has defined the Principle of Parsimony as "Frusta fit per plura, quod fieri potest per pauciora", i.e. it is vain to do with more what can be done with less (Upton and Cook, 2008). Founded on that idea and considering the conceptual analogy proposed by the procedure FCAUP, it is acceptable that approach 3 (partial double inspection) is in fact a suitable option during conformity assessments if compared to approach 1 (no double inspection) and approach 2 (100\% of double inspection). In general, this understanding is correct once bearing in mind both technical and operational characteristics, as well as economic aspects.

\section{Conclusions}

The main contribution of the present research is the introduction of a novel procedure called as FCAUP, a five-stage guide for performing conformity assessment based on an analogy with Heisenberg's
Uncertainty Principle. The Uncertainty Principle refers to physical phenomena of position and momentum of particles at subatomic scales, whereas the conformity assessment deals with some level of uncertainty related to activities to demonstrate the fulfillment of specified requirements by products, processes, systems, personnel or bodies.

In this conceptual analogy, the uncertainty regarding particle-like records (PLR) can be preliminarily reduced by means of double inspections on position (objective information) by considering purposive sampling based on qualitative trade-off. With respect to the uncertainty related to wave-like records (WLR), this can be mitigated via verification of momentum, i.e. subjective and/or interpretative evaluations. A quantum-like behavior regarding these records may arise due to qualitative nuances and, in this case, additional appraisals are required. The comprehension of this possible quantum-like scenario is a decisive factor in the execution of the structured activities recommended by FCAUP.

Following the discussions presented so far, it can be concluded that a partial double inspection is more appropriate than a complete double inspection or no double inspection at all, and this understanding is correct by considering technical, operational and economic aspects. Furthermore, for all situations in which there is an extensive review of records during conformity assessment activities, no more than $50 \%$ of double inspection is enough to obtain useful and reliable results, even in situations in which the degree of trust in conformity is very low. However, it is important to emphasize that only the implementation of double inspection cannot eliminate all uncertainty inherent to the conformity assessment process, but, whenever applicable, double inspection can be considered an effective assessment tool.

To sum up, it is already possible to assume that the method FCAUP (Framework for Conformity Assessment inspired by the Uncertainty Principle) introduced herein can be satisfactorily used in several categories of conformity assessment, mainly in first-party assessments, by considering applicable amendments and adaptations. A framework like this is an appropriate tool to improve both technical and operational activities of conformity assessment in manufacturing industries and in many other areas as previously mentioned, but it is also helpful in terms of strategic planning, since it supports decision makers in their evaluations related to quality management. Nevertheless, further investigations on actual conformity assessments should be conducted in order to achieve a full validation of FCAUP.

\section{Acknowledgments}

The authors would like to thank the Fundação de Apoio à Universidade de São Paulo (FUSP) and the anonymous reviewers for their critical analyses, valuable comments and helpful suggestions. 


\section{References}

Assalim, L., Almeida, M.F.L., 2013. Conformity assessment as a tool for organizational learning in large engineering and construction projects. J. Technol. Manag. Innovat. 8, 37-48.

Carobbi, C., Pennecchi, F., 2016. Bayesian conformity assessment in presence of systematic measurement errors. Metrologia 53, S74-S80.

Cho, Y.S., Jung, J.Y., Linderman, K., 2017. The QM evolution: behavioral quality management as a firm's strategic resource. Int. J. Prod. Econ. 191, 233-249.

Fedak, W.A., Prentis, J.J., 2009. The 1925 Born and Jordan paper "On quantum mechanics". Am. Assoc. Phys. Teach. 77, 128-139.

Fitts, D.D., 2002. Principles of Quantum Mechanics: as Applied to Chemistry and Chemical Physics. Cambridge University Press.

Gerolamo, M.C., Poltronieri, C.F., Yamada, T.T., Cintra, A.L.B., 2014. Quality management: how do brazilian companies use it? Procedia-Soc. Behav. Sci. 143, 995-1000.

Griffiths, D.J., 2011. Introduction to Quantum Mechanics. Prentice Hall, Upper Saddle River, New Jersey.

International Organization for Standardization, 2015. ISO 9000:2015: Quality Management Systems - Fundamentals and Vocabulary. Genève, Switzerland.

International Organization for Standardization, International Electrotechnical

Commission, 2004a. ISO/IEC 17000:2004: Conformity Assessment - Vocabulary and General Principles. Genève, Switzerland.

International Organization for Standardization, International Electrotechnical Commission, 2004b. ISO/IEC GUIDE 60:2004: Conformity Assessment - Code of Good Practice. Genève, Switzerland.

Jijnasu, V., 2016. The uncertainty principle - a simplified review of the four versions. Stud. Hist. Philos. Mod. Phys. 55, 62-71.

Juran, J.M., Godfrey, A.B.(Org.), 1999. Juran's Quality Handbook, fifth ed. McGraw-Hill, Inc, New York.

Kuselman, I., Pennecchi, F., Silva, R.J.N.B., Hibbert, D.B., 2017. Conformity assessment of multicomponent materials or objects: risk of false decisions due to measurement uncertainty - a case study of denatured alcohols. Talanta 164, 189-195.

Li, D., Zhao, Y., Zhang, L., Chen, X., Cao, C., 2017. Impact of quality management on green innovation. J. Clean. Prod. https://doi.org/10.1016/j.jclepro.2017.09.158. available online 22/September/2017.

Liepina, R., Lapina, I., Mazais, J., 2014. Contemporary issues of quality management: relationship between conformity assessment and quality management. Procedia-Soc. Behav. Sci. 110, 627-637.

Lüth, S., Ivanova, A., Kempka, T., 2015. Conformity assessment of monitoring and simulation of $\mathrm{CO}_{2}$ storage: a case study from the Ketzin pilot site. Int. J. Greenh. Gas Contr. 42, 329-339.

MarsalL-Llacuna, M.L., 2017. The standards evolution: a pioneering Meta-standard framework architecture as a novel self-conformity assessment and learning tool. Comput. Stand. Interfac. 000, 1-10.

Pendrill, L.R., 2014. Using measurement uncertainty in decision-making and conformity assessment. Metrologia 51, S206-S218.

Rusu, C., 2016. From quality management to managing quality. Procedia-Soc. Behav. Sci. 221, 287-293.

Shankar, R., 1994. Principles of Quantum Mechanics, second ed. Plenum Press, New York.

Simon, A., Bernardo, M., Karaptrovic, S., Casadesús, M., 2011. Integration of standardized environmental and quality management systems audits. J. Clean. Prod. 19, 2057-2065.

Siva, V., Gremyr, I., Bergquist, B., Garvare, R., Zobel, T., Isaksson, R., 2016. The support of Quality Management to sustainable development: a literature review. J. Clean. Prod. 138, 148-157.

Suwandej, N., 2015. Factors influencing total quality management. Procedia-Soc. Behav Sci. 197, 2215-2222.

Theodorou, D., Zannikos, F., 2014. The use of measurement uncertainty and precision data in conformity assessment of automotive fuel products. Measurement 50, $141-151$.

Upton, G., Cook, I., 2008. Dictionary of Statistics, second ed. Oxford University Press.

Wiengarten, F., Pagell, M., 2012. The importance of quality management for the success of environmental management initiatives. Int. J. Prod. Econ. 140, 407-415.

Zeng, J., Zhang, W., Matsui, Y., Zhao, X., 2017. The impact of organizational context on hard and soft quality management and innovation performance. Int. J. Prod. Econ. $185,240-251$.

Zozor, S., Portesi, M., Vignat, C., 2008. Some extensions of the uncertainty principle. Physica A 387, 4800-4808. 